\title{
Dynamic Distortion Control for 3-D Embedded Wavelet Video over Multiuser OFDM Networks
}

\author{
Guan-Ming Su, Zhu Han, Min Wu, and K.J. Ray Liu \\ Department of Electrical and Computer Engineering, University of Maryland, College Park, MD, USA. \\ Email: \{gmsu, hanzhu, minwu, kjrliu\}@eng.umd.edu
}

\begin{abstract}
In this paper, we propose a system to transmit multiple 3-D embedded wavelet video programs over downlink multi-user OFDM. We consider the fairness among users and formulate the problem as minimizing the users' maximal distortion subject to power, rate, and subcarrier constraints. By exploring frequency, time, and multiuser diversity in OFDM and flexibility of 3D embedded wavelet video codec, the proposed algorithm can achieve fair video qualities among all users. Compared to a scheme similar to the current multiuser OFDM standard (IEEE 802.11a), the proposed scheme outperforms $1 \sim 5 \mathrm{~dB}$ on the worst received PSNR among all users and has much smaller PSNR deviation.
\end{abstract}

\section{INTRODUCTION}

Orthogonal frequency division multiplexing (OFDM) is a promising modulation scheme for broadband system such as wireless video. Since different video scenes have different content complexities, at a given bit rate, some scenes may have unnecessarily high perceptual quality, while others may have low perceptual quality. On the other hand, if the users pay the same price for certain video quality, the received qualities for these users should be similar. How the server jointly allocates the system resource to each stream to provide comparable perceptual quality is an important fairness issue [1], [2], which we will investigate in this work. As we shall see, a multiuser OFDM can provide frequency, time, and multiuser diversity to achieve fair and high video qualities among all users.

To transmit video over wireless channel, a system should be able to adjust the source bit rate according to the varying channel conditions. A highly scalable video codec is desired since it provides flexibility and convenience in reaching the desired visual quality or the desired bit rate. The Fine Granularity Scalability (FGS) coding and Fine Granular Scalability Temporal (FGST) coding in the MPEG-4 video coding standard can provide high flexibility, but their overall qualities are worse than the non-scalable coding result and still have a non-scalable base layer. The development of 3-D subband video coding provides an alternative to compress video with full scalability. Unlike the block matching motion compensated video codec (such as H.263 and MPEG-4), the 3-D embedded wavelet video (EWV) coding in [3] explores the spatiotemporal redundancies via 3-D wavelet transform. Extending the bit allocation ideas from EBCOT algorithm [4], EWV outperforms MPEG-4 for sequences with low or moderate motion and has comparable performance to MPEG-4 for most high-motion sequences. Therefore, we adopt EWV as a basis for the source coding part in our work.

A system transmitting a single video program is a special case to our multiuser problem, such as the work in [5] concerning the transmission of H.263 video over OFDM. Zheng and Liu [6] proposed a parallel transmission framework for layered video to minimize the received distortion. Systems transmitting layered image/video over space-time coded OFDM were proposed in [7], [8]. A joint source-channel bit allocation scheme for MPEG-4 FGS video over OFDM was studied in [9]. The video broadcasting system in [10] minimizes the maximal distortion received by all users. Extending to the scenario of supporting multiple video programs, the authors in [11] examined the subcarrier assignment problem. However, a greedy assignment achieving the minimal overall received distortion may lead to unfairness as some users may receive extremely good quality and some others receive extremely bad quality. In this paper, we design a multiuser wireless video system providing fairness among users. We formulate the problem as an optimization problem and provide a fast and suboptimal solution. The experimental results show that the proposed scheme can achieve more fair and better video quality than a scheme similar to the current multiuser OFDM standard (IEEE 802.11a).

This paper is organized as follows. The 3-D EWV and the system architecture are described in Section II. In Section III, we formulate this system as a min-max problem and present the proposed algorithm. The simulation results are presented in Section IV and conclusions are drawn in Section V.

\section{SySTEM DESCRIPTION}

In this section, we first review the 3-D embedded wavelet video codec and then introduce our proposed system of sending multiple video programs over OFDM.

a) 3-D EWV Codec: The EWV encoder consists of four parts: 3-D wavelet transform, quantization, bit plane arithmetic coding, and rate-distortion (R-D) optimization. At the first stage, we collect a group of frames (GOF) as an encoding unit and apply 1-D dyadic temporal decomposition to obtain temporal subbands. The 2-D spatial dyadic decomposition is applied in each temporal subband to obtain wavelet spatiotemporal subbands (or "subband" in short). At the second stage, a uniform quantizer is used for all wavelet coefficients in all subbands. At the third stage, bit plane arithmetic coding is applied to each subband. Every bit plane is encoded into three coding passes except only one pass for the most significant bit plane. The EWV decoder can decode the truncated bitstream containing an integer number of coding passes in each subband. The more consecutive coding passes of each subband a receiver receives, the higher decoded video quality we have. The coding passes among all subbands can be further grouped into several quality layers such that the received video quality can be refined progressively. At the last stage, the encoder determines the 
coding passes and the subbands to achieve the desired quality or rate.

To maintain the coding efficiency, the R-D curve in each subband should be convex [4]. Some coding passes in a subband cannot serve as feasible truncation points to maintain the convexity and they will be pruned from the truncation point list. To facilitate the discussion, we call all the coding passes between two truncation points as a coding pass cluster.

Consider now there are a total of $B$ subbands for the $k^{\text {th }}$ user and the subband $b$ has $T_{k}^{b, \max }$ truncation points. We can measure the increased rate and the corresponding decreased mean squared distortion between the $(t-1)^{t h}$ and $t^{t h}$ truncation points in subband $b$ for the $k^{t h}$ user and denote them as $\Delta r_{t, b, k}$ and $\Delta d_{t, b, k}$, respectively. We divide the whole duration for transmitting a total of $L$ quality layers into $L$ transmission intervals with equal length. The $l^{\text {th }}$ quality layer is sent at the $l^{t h}$ transmission interval. The received distortion $D_{k}^{l}$ and the rate $R_{k}^{l}$ for quality layers $O$ to $l$ can be expressed as:

$$
\begin{aligned}
& D_{k}^{l}=D_{k}^{\max }-\sum_{b=0}^{B-1} \sum_{t=0}^{T_{k}^{b, l}-1} \Delta d_{t, b, k}, \\
& R_{k}^{l}=\sum_{q=0}^{l} \Delta R_{k}^{q},
\end{aligned}
$$

where $D_{k}^{\max }$ is the distortion without having any coding pass cluster, and

$$
\Delta R_{k}^{l}=\sum_{b=0}^{B-1} \sum_{t=T_{k}^{b, l-1}}^{T_{k}^{b, l}-1} \Delta r_{t, b, k},
$$

$T_{k}^{b, l}$ is the total number of coding pass clusters of subband $b$ in the quality layers 0 to $l$, which satisfies:

$$
0 \leq T_{k}^{b, l-1} \leq T_{k}^{b, l} \leq T_{k}^{b, \max }, \quad \forall b \quad \text { and } \quad 0<l<L .
$$

Define the number of coding pass clusters for subband $b$ in quality layer $l$ as $\Delta T_{k}^{b, l}=T_{k}^{b, l}-T_{k}^{b, l-1}$ and for all subbands

$$
\Delta \mathbf{T}_{k}^{l}=\left[\Delta T_{k}^{0, l}, \Delta T_{k}^{1, l}, \ldots, \Delta T_{k}^{B-1, l}\right] .
$$

We also define a matrix $\Delta \mathbf{T}^{l}$ whose $k^{t h}$ row contains $\Delta \mathbf{T}_{k}^{l}$. Thus, in each transmission interval $l$, the source coding part of our system determines the vector of coding pass cluster numbers $\Delta \mathbf{T}_{k}^{l}$ for each user and packetize them as a quality layer subject to the rate constraint $\Delta R_{k}^{l} \leq R_{k}$, where $R_{k}$ is allocated overall transmission rate for user $k$ at interval $l$.

b) Multiuser OFDM: We consider a downlink scenario of a single-cell multiuser OFDM system. The system has $N$ subcarriers and there are $K$ users randomly located within the cell. Each subcarrier has a bandwidth of $W$. We use an indicator $a_{k n} \in\{0,1\}$ to represent whether the $n^{\text {th }}$ subcarrier is assigned to user $k$. Note that in a single-cell OFDM system, each subcarrier can be assigned to at most one user, i.e. $\sum_{k=0}^{K-1} a_{k n} \in$ $\{0,1\}, \forall n$. The overall subcarrier-to-user assignment can be represented as a matrix $\mathbf{A}$ with $[\mathbf{A}]_{k n}=a_{k n}$. Denote $r_{k n}$ as the $k^{t h}$ user's transmission rate in the $n^{\text {th }}$ subcarrier. The rate requirement is $R_{k}=\sum_{n=0}^{N-1} a_{k n} r_{k n}$. Define the rate allocation matrix $\mathbf{R}$ as $[\mathbf{R}]_{k n}=r_{k n}$.

We assume slow fading channel such that the channel gain is stable within each transmission interval. For simplicity, the
TABLE I

REQUIRED SNR FOR BER $=10^{-6}$ OF DIFFERENT ADAPTIVE MODULATION AND CONVOLUTIONAL CODING RATES [12]

\begin{tabular}{|c|c|c|c|}
\hline Rate & Modulation & $\begin{array}{c}\text { Convolutional } \\
\text { Coding Rate }\end{array}$ & $\begin{array}{c}\text { SNR }(\mathrm{dB}) \text { for } \\
\text { BER } \leq 10^{-6}\end{array}$ \\
\hline $1 \mathrm{~W}$ & QPSK & $1 / 2$ & 4.65 \\
$1.33 \mathrm{~W}$ & QPSK & $2 / 3$ & 6.49 \\
$1.5 \mathrm{~W}$ & QPSK & $3 / 4$ & 7.45 \\
$1.75 \mathrm{~W}$ & QPSK & $7 / 8$ & 9.05 \\
$2 \mathrm{~W}$ & 16QAM & $1 / 2$ & 10.93 \\
$2.66 \mathrm{~W}$ & 16QAM & $2 / 3$ & 12.71 \\
$3 \mathrm{~W}$ & 16QAM & $3 / 4$ & 14.02 \\
$3.5 \mathrm{~W}$ & 16QAM & $7 / 8$ & 15.74 \\
$4 \mathrm{~W}$ & 64QAM & $2 / 3$ & 18.50 \\
$4.5 \mathrm{~W}$ & 64QAM & $3 / 4$ & 19.88 \\
$5.25 \mathrm{~W}$ & 64QAM & $7 / 8$ & 21.94 \\
\hline
\end{tabular}

channel parameters from different subcarrier of different users are assumed to have been perfectly estimated, and the channel information is reliably fed back from mobiles users to the base station without delay. Denote $\Gamma_{k n}$ as the $k^{\text {th }}$ user's signal to noise ratio (SNR) at the $n^{\text {th }}$ subcarrier as:

$$
\Gamma_{k n}=P_{k n} G_{k n} / \sigma^{2} \text {, }
$$

where $G_{k n}$ is the subcarrier channel gain and $P_{k n}$ is the transmitted power for the $k^{t h}$ user in the $n^{t h}$ subcarrier. The thermal noise power for each subcarrier is assumed to be the same and represented as $\sigma^{2}$. Further, let $[\mathbf{G}]_{k n}=G_{k n}$ be the channel gain matrix and $[\mathbf{P}]_{k n}=P_{k n}$ the power allocation matrix. For downlink system, the overall system power is bounded by $P_{\max }$, i.e., $\sum_{k=0}^{K-1} \sum_{n=0}^{N-1} a_{k n} P_{k n} \leq P_{\max }$.

We also assume that with appropriate channel modulation and coding, the distortion introduced by the channel on the received video is negligible. This is possible when bit error rate (BER) of the channel transmission is kept low. Adaptive modulation and adaptive channel coding provide each user with the ability to adjust each subcarrier's data transmission rate $r_{k n}$, according to the channel condition while the desired BER is met. We focus our attention to MQAM modulation and convolutional codes as they provide high spectrum efficiency and strong forward error protection, respectively. The required SNRs at $\mathrm{BER}=10^{-6}$ for different modulations and convolutional coding rates using bit interleaved coded modulation (BICM) are listed in Table I [12]. Thus, for each rate allocation matrix $\mathbf{R}$, the power allocation matrix $\mathbf{P}$ should maintain the $\mathrm{SNR} \Gamma_{k n}$ larger than the corresponding value in Table I.

c) Video over OFDM: The system block diagram is shown in Fig. 1. The server buffers each user's incoming video frames in the user's frame buffer. For each video, a GOF with $H$ frames are moved to wavelet video encoder and compressed as a coding pass bit stream. The selected coding pass clusters will be transmitted during the next GOF transmission time of $H / F$ second long, where $F$ is the video sampling rate. The resource allocator obtains the R-D information from the source coder and the channel condition information from the channel estimator. In each transmission interval, the allocator performs resource optimization for distortion management: at the source coding subsystem, a selected number of coding pass clusters are grouped into a quality layer and passed to the transmission system; at the OFDM subsystem, the data to be transmitted are loaded to different subcarriers at a controlled amount of power. 


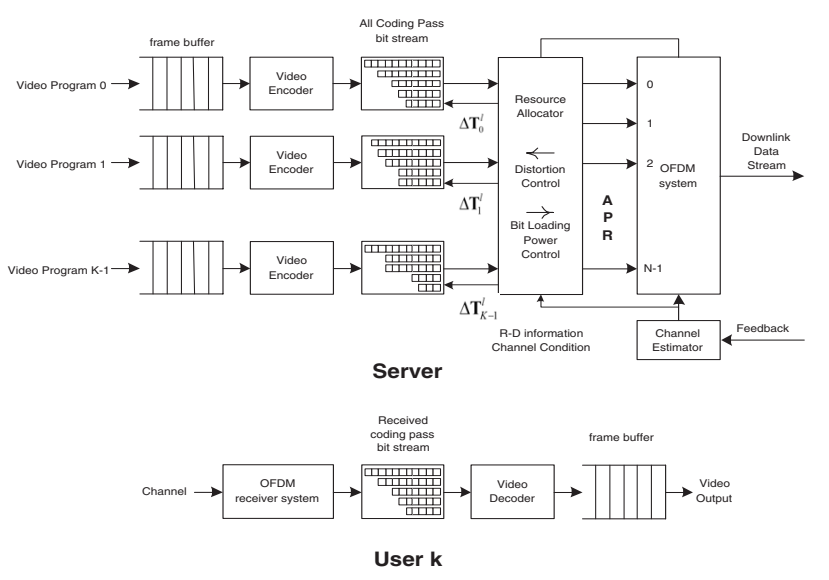

Fig. 1. System block diagram

For each mobile user, an OFDM receiver buffers the received data until the end of the current GOF transmission time. Then, those data are decoded by a wavelet video decoder and the decoded frames are sent for display.

\section{Optimization in Resource Allocator}

a) Problem Formulation: We formulate the problem as a min-max optimization problem to minimize the maximal (weighted) end-to-end distortion among all users to ensure fairness. We assume the channel is slow fading and the channel coherent interval is no less than the transmission interval. Since we only know the channel conditions provided by the channel estimator in the near future, such as up to the next transmission interval, we need to break down our problem into a sequential optimization problem and solve each problem in each transmission interval. At the beginning of the $l^{\text {th }}$ transmission interval, according to the channel information, the resource allocator solves the following optimization problem:

$$
\begin{aligned}
& \min _{\mathbf{A}, \mathbf{R}, \Delta \mathbf{T}^{l}} \max _{k} w_{k} \cdot f\left(D_{k}^{l}\right) \\
& \text { subject to }\left\{\begin{array}{l}
\sum_{k=0}^{K-1} a_{k n} \leq 1, a_{k n} \in\{0,1\}, \forall n ; \\
0 \leq \Delta R_{k}^{l} \leq \sum_{n=0}^{N-1} a_{k n} r_{k n}, \forall k ; \\
\sum_{k=0}^{K-1} \sum_{n=0}^{N-1} a_{k n} P_{k n} \leq P_{\max } ;
\end{array}\right.
\end{aligned}
$$

where $w_{k}$ is the quality weight factor and $f(\cdot)$ the perceptual distortion function. The first constraint is the subcarrier assignment constraint, the second one is the rate constraint for each user, and the third one is the maximal power constraint. Note that the system can provide differentiated service by setting $\left\{w_{k}\right\}$ to different values according to the quality levels requested by each user. As a proof-of-concept, we consider the case of $w_{k}=1, \forall k$, and $f\left(D_{k}^{l}\right)=D_{k}^{l}$ for providing uniform quality among all users. Our solution can be extended to other quality weight factors and quality functions.

b) Solution: To solve the multi-variable mixed integer optimization problem in (7), we propose a three-step algorithm shown in Fig. 2. First, the resource allocator obtains GOF R-D function of all unsent coding pass clusters for each user. The operational R-D function specifies the required data rates for reducing a certain amount of distortion. Second, the resource allocator checks the highest rate increase that the OFDM system

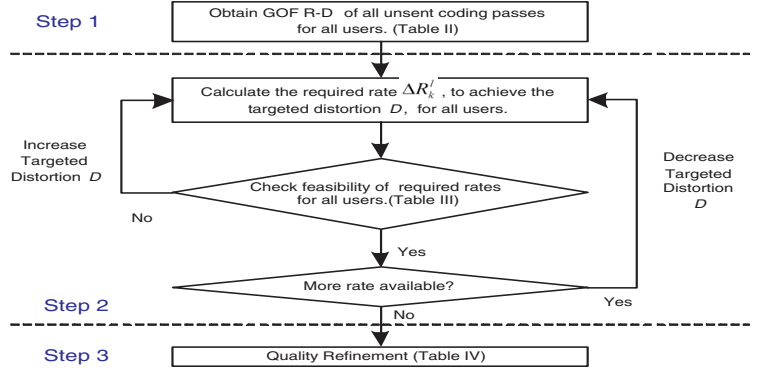

Fig. 2. Flow chart of the proposed algorithm

TABLE II

GOF R-D USED IN EACH TRANSMISSION INTERVAL

\begin{tabular}{|l|}
\hline a) Sort all $\left\{\lambda_{t, b, k}\right\}$ for all $t \geq T_{k}^{b, l-1}$ in a decreasing order for user $k$ \\
\hline b) For each $(t, b)$ for user $k$, we have indices \\
$I_{k}(t, b) \in\left\{1,2, \ldots, M_{k}^{l}\right\}$ and $I_{k}^{-1}(m) \in\{(t, b)\}$ \\
s.t. $\lambda_{i, b, k}>\lambda_{j, c, k}$ for $I_{k}(i, b)<I_{k}(j, c)$ \\
\hline c) Set $D_{k}^{l}[0]=D_{k}^{l-1}$ and $R_{k}^{l}[0]=0$ \\
For $m=1, \ldots, M_{k}^{l}$ \\
$\quad D_{k}^{l}[m]=D_{k}^{l}[m-1]-\left|\Delta d_{I_{k}^{-1}(m), k}\right|$ \\
$R_{k}^{l}[m]=R_{k}^{l}[m-1]+\Delta r_{I_{k}^{-1}(m), k}$ \\
\hline
\end{tabular}

can support. Finally, we perform a round of quality refinement by utilizing unused bandwidth. We explain the details of these three steps below.

In Step 1, we obtain the GOF R-D of unsent coding pass clusters for each user. Define the distortion-to-length slope for each coding pass cluster as follows:

$$
\lambda_{t, b, k}=\left|\Delta d_{t, b, k}\right| / \Delta r_{t, b, k} .
$$

Suppose there are $M_{k}^{l}$ unsent coding pass clusters for user $k$. We sort all distortion-to-length slope of all unsent coding pass clusters $\left(\lambda_{t, b, k}\right.$, where $\left.T_{k}^{b, l-1} \leq t\right)$ in a decreasing order and obtain the corresponding mapping indices $I_{k}(t, b)$. The sorting order represents the transmitting priority: if a coding pass cluster with index $m$ is sent, all coding pass clusters with index value smaller than $m$ must be sent. We use this sorting order to obtain the operational R-D curve $\left(D_{k}^{l}[m], R_{k}^{l}[m]\right)$ for quality layer $l$. The details are presented in Table II. The complexity of this step for each user is $O\left(M_{\hat{k}}^{l} \log \left(M_{\hat{k}}^{l}\right)\right)$ due to the sorting.

In Step 2, the goal is to minimize the maximal distortion to the lowest level with the corresponding data rate supportable by the OFDM subsystem. By checking the R-D curves obtained in Step 1, the resource allocator can calculate the required transmission rates $\Delta R_{k}^{l}$ to obtain the same targeted distortion $D$ for all users. Then the resource allocator checks if the requested rates are feasible for OFDM in the current channel conditions. If the requested rates are feasible, the resource allocator tries to further decrease the targeted distortion by increasing the requested rates. Otherwise, the resource allocator increases the targeted distortion to reduce the requested rates and checks the feasibility again. A bisection search algorithm is developed to find the minimal distortion $D$ that the OFDM subsystem can support. Finally, the corresponding information of bit loading and power control is sent to the OFDM subsystem.

The feasibility of the requested rates depends on two factors. One factor is that the OFDM subsystem should be able to transmit the requested rates $\left\{\Delta R_{k}^{l}\right\}$ for all users; and the other 
TABLE III

OFDM Resource Allocation AND FeAsibility CHECK

\begin{tabular}{l}
\hline a) Initialization: Get $\Delta R_{k}^{l}, \forall k$ and set $\mathbf{A}=0, \mathbf{R}=0$, and $\mathbf{P}=0$. \\
\hline b) Minimal Rate Assignment: \\
While $\sum_{n=0}^{N-1} r_{k n} a_{k n} \geq \Delta R_{k}^{l}, \forall k$ not satisfied \\
1) Find arg $\max _{k, n}[\mathbf{G}]_{k n}$. \\
2) Assign subcarrier $n$ to user $k$. Set $G_{k n}=0, \forall k$. Waterfill all \\
subcarriers of user $k$ to minimize power with rate $\Delta R_{k}^{l}$. \\
3) If the requested rate of user $k$ is achieved, set $G_{k n}=0, \forall n$. \\
4) If no subcarriers left and not all requested rates are satisfied, \\
report infeasibility and exit. \\
c) Power Reduction: \\
While there are subcarriers left \\
1) Assign user $k$ who has the highest average power per subcarrier \\
with a remaining subcarrier having the largest $G_{k n}$ for user $k$. \\
2) Minimize the transmission power among the subcarrier set \\
assigned to this user. \\
Calculate the overall power. If greater than $P_{m a x}$, report infeasibility; \\
Otherwise, report feasibility and calculate $\mathbf{A}, \mathbf{R}$, and $\mathbf{P}$.
\end{tabular}

factor is that the overall transmitted power cannot exceed $P_{\max }$. We develop a heuristic algorithm shown in Table III to allocate the bits and power to satisfy the rate constraint first and then the power constraint. First, the subcarrier assignment matrix $\mathbf{A}$, the rate assignment matrix $\mathbf{R}$, and the power assignment matrix $\mathbf{P}$ are initialized to zeros. Second, the system tries to satisfy the requested rates. In each round, we allocate an unassigned subcarrier to a user. If $G_{k n}$ has the maximal value in current $\mathbf{G}$, we assign subcarrier $n$ to user $k$ and set $G_{k n}=0 \forall k$ to prevent this subcarrier from being assigned again. We then determine the modulation schemes and the coding rates for all subcarriers currently allocated to user $k$ such that the requested data rate can be accommodated and the power is minimized. This can be implemented by the well-known waterfilling algorithm with Table I and (6). If the requested rate can be allocated, user $k$ is removed from future assignment list in this step by assigning $G_{k n}=0, \forall n$. We repeat this process until all users' requested rates are satisfied. If all subcarriers are already assigned and not all requested rates are achieved, infeasibility is reported and the resource allocator has to reduce the requested rates. Next, we minimize the overall transmitted power by assigning the remaining subcarriers. In each round, we assign the user with the highest average power per subcarrier with a remaining subcarrier having the largest channel gain for this user. Then we minimize the transmission power among the subcarrier set assigned to this user. The above assignment is repeated until no subcarrier is left. We calculate the overall transmitted power and if it is greater than $P_{\max }$, infeasibility is reported. Otherwise, we calculate $\mathbf{A}, \mathbf{R}$, and $\mathbf{P}$ for OFDM subsystem. Since the required power for each user in each subcarrier can be pre-calculated, the complexity of checking feasibility in each iteration is $O(N)$. The overall number of iteration is bounded by the bisection search, which is typically fewer than 20 in our experiment.

In Step 3, we further refine the video quality. Due to the discrete rate information of both video source and OFDM subsystems, the rate assignment generated from Step 2 is generally larger than the requested rate. We may be able to add more coding pass clusters with the extra bandwidth. We search all unsent coding pass clusters that follow the currently selected truncation points and pick those with rates smaller
TABLE IV

QUALITY REFINEMENT

\begin{tabular}{l}
\hline a) Calculate unused rate $U R_{k}^{l}=\sum_{n=0}^{N-1} a_{k n} r_{k n}-\Delta R_{k}^{l}$ \\
\hline b) While $U R_{k}^{l} \geq 0$ \\
1) Search the coding pass clusters set, $S$, whose element \\
satisfies $\Delta r_{t, b, k} \leq U R_{k}^{l}$ and $t=T_{k}^{b, l}$ in all subbands \\
2) If set $S$ is empty, leave the loop \\
3) Select the coding pass cluster, $\hat{b}$, with largest $\lambda_{t, b, k}$ in set $S$ \\
4) Update $T_{k}^{\hat{b}, l}=T_{k}^{\hat{b}, l}+1$ and $U R_{k}^{l}=U R_{k}^{l}-\Delta r_{t, \hat{b}, k}$
\end{tabular}

than the unused bandwidth. The coding pass cluster with the largest distortion-to-length slope is selected for transmission during current transmission interval. The system updates the unused bandwidth and unsent coding pass clusters, and repeats this procedure until there is no coding pass cluster with size smaller than the unused bandwidth. The complexity to search a feasible coding pass cluster is $O(B)$.

\section{Simulation Results}

The simulations are set up as follows. The OFDM system has 32 subcarriers over a total $1.6 \mathrm{MHz}$ bandwidth. The average delay spread $\mathrm{RMS}=3 \times 10^{-7} \mathrm{~s}$. An additional $5 \mu \mathrm{s}$ guard interval is used to avoid inter-symbol-interference due to channel delay spread. This results in a total block length as $25 \mu \mathrm{s}$ and a block rate as $40 \mathrm{k}$ per second. The transmission interval is $33.33 \mathrm{~ms}$. The mobile is uniformly distributed within the cell with radius from $10 \mathrm{~m}$ to $50 \mathrm{~m}$. The noise power is $10^{-9}$ Watts, and the maximal transmitted power is 0.1 Watts. The Doppler frequency is $10 \mathrm{~Hz}$ and the propagation loss factor is 1.8 [13].

The video sampling rate is 30 frames per second. The GOF size is 16 frames and each GOF is encoded using the codec [3] with 4-level temporal decomposition. We use a four-user system with uniform quality among users to demonstrate the proposed algorithm. User 0 to 3 receive one GOF from video sequence, Foreman, Hall Monitor, Mother and daughter, and Silent, respectively. Fig. 3 shows the maximal, minimal, and average MSE received among all users in each transmission interval. Due to the discrete and convex R-D function, discrete channel transmission rate, and quality refinement (Step 3), there is a gap between the best and worst received quality at the first few transmission intervals. However, the received quality is refined at the following intervals and the quality gap among users is reduced through (7). At the end, we achieve the required fair quality among all users. The corresponding subcarrier assignment in each transmission interval is shown in Fig. 4. As the source coding rate of each user is allocated in different time and frequency slots according to the channel conditions and source characteristics, the diversity of frequency, time, and multiuser is effectively exploited.

We compare the proposed algorithm with a TDMA-like algorithm, which is a scheduling method similar to IEEE 802.11a. Instead of allowing subcarriers in a transmission interval to be allocated among multiple users, the TDMA algorithm assigns all subcarriers in one transmission interval to only one user whose current distortion is the largest. We concatenate 15 classic CIF video sequences (Akiyo, Bus, Coastguard, Container, Flower, Foreman, Hall Monitor, Highway, Mobile, Mother and daughter, MPEG4 news, Paris, Silent, Tempete, and Waterfall) 


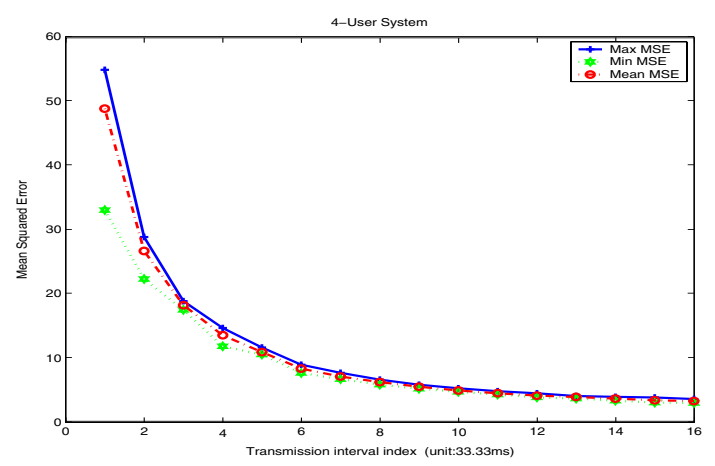

Fig. 3. Distortion improvement in each transmission interval

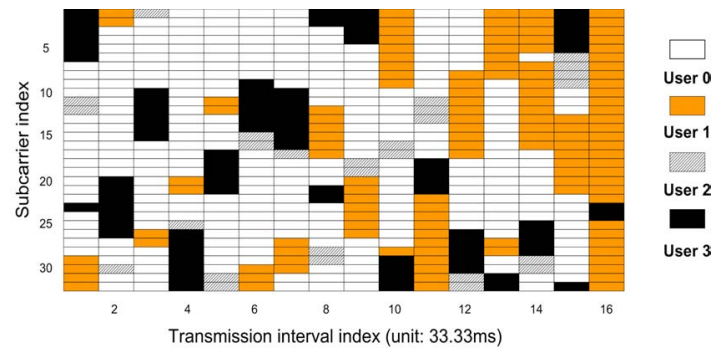

Fig. 4. Subcarrier assignment in each transmission interval. The system assigns more subcarriers to user 0 at most intervals due to the required rate of video sequence 0 to achieve the same quality is higher than other sequences.

to form a basic testing sequence of 4064 frames. The video for the $k^{\text {th }}$ user is 160 frames long and from frame $256 \times(k-1)+1$ to frame $256 \times(k-1)+160$ of the testing sequence. Fig. 5 shows the average value of the worst received PSNR among all users from 10 different terminals' locations for different number of users in the system. We can see that the proposed algorithm can maximize the minimal PSNR better than the TDMA scheme. There is about $1 \mathrm{~dB}$ gain when the number of users is less than 8. This is because the proposed scheme employs additional diversity in frequency and multiuser. The performance gain becomes higher when the number of users is increased owing to multiuser diversity.

To demonstrate the distribution of the received quality for these two algorithms, we plot in Fig. 6 the histogram of the PSNR deviation (defined as the difference between the received PSNR and the average PSNR of all users for each video frame interval). As we can see, the dynamic range of PSNR deviation of the TDMA algorithm is about $20 \mathrm{~dB}$ while that of the proposed algorithm is only about $6 \mathrm{~dB}$. We also notice that the PSNR deviation of the TDMA algorithm has two peaks around $\pm 5 \mathrm{~dB}$, which implies the quality is either very good or very bad. On the other hand, the proposed algorithm has much improved fairness providing consistent quality among all users.

\section{CONCLUSION}

In this paper, we have constructed a multiuser wireless video system sending 3-D wavelet video over OFDM. To achieve fair quality among all users, we formulated this system as a minimax problem and proposed an effective algorithm by leveraging the frequency, time, and multiuser diversity of the OFDM system and the scalability of 3-D embedded wavelet video codec. The

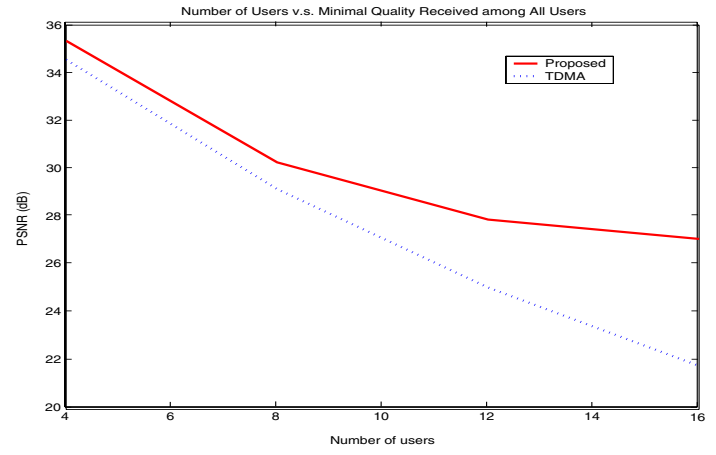

Fig. 5. The worst quality received among all users v.s. the number of users
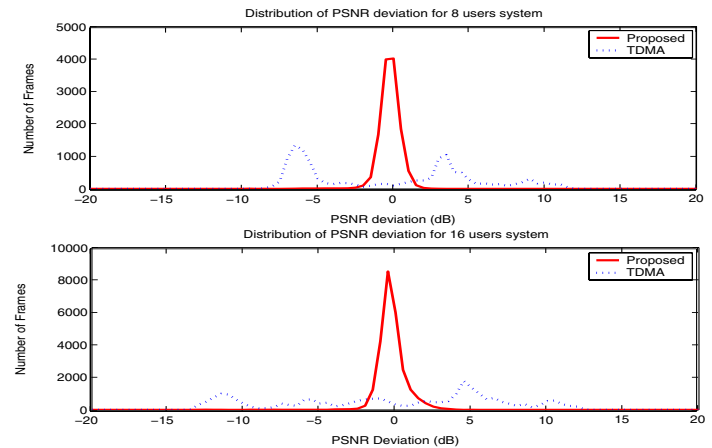

Fig. 6. Histogram of PSNR deviation from the average PSNR

worst PSNR received among all users of the proposed algorithm outperforms TDMA algorithm $1 \sim 5 \mathrm{~dB}$ with much smaller PSNR deviation. Thus, the proposed scheme is a promising building block for broadband multimedia communication.

\section{REFERENCES}

[1] L. Wang and A. Vincent, "Bit allocation and constraints for joint coding of multiple video programs", IEEE CSVT, pp. 949-959, Sep. 1999.

[2] X.M. Zhang, A. Vetro, Y.Q. Shi, and H. Sun, "Constant quality constrained rate allocation for FGS-coded videos", IEEE CSVT, pp. 121-130, Feb. 2003.

[3] J. Hua, Z. Xiong, and X. Wu, "High-performance 3-D embedded wavelet video (EWV) coding," IEEE MMSP Workshop, 2001.

[4] D. S. Taubman, "High performance scalable image compression with EBCOT," IEEE Trans. Image Processing, pp.1158-1170, Jul. 2000.

[5] P.J. Cherriman, T. Keller, and L. Hanzo, "Orthogonal frequency-division multiplex transmission of H.263 encoded video over highly frequencyselective wireless networks," IEEE CSVT, pp.701-712, Aug. 1999.

[6] H. Zheng and K.J.R. Liu, "Robust image and video transmission over spectrally shaped channels using multicarrier modulation," IEEE Transactions on Multimedia, vol.1, no. 1, pp.88-103, Mar. 1999.

[7] Y. Sun, Z. Xiong, and X. Wang, "Scalable image transmission over differentially space-time coded OFDM systems," IEEE Globalcom, 2002.

[8] C.-H. Kuo, C.-S. Kim, and C.-C.J. Kuo, "Robust video transmission over wideband wireless channel using space-time coded OFDM systems," IEEE WCNC, vol.2, pp.931-936, Mar. 2002.

[9] J. Xu, Q. Zhang, W. Zhu, X.-G. Xia, and Y.-Q. Zhang, "Optimal joint source-channel bit allocation for MPEG-4 fine granularity scalable video over OFDM system," ISCAS v.2, May 2003.

[10] Z. Ji, Q. Zhang, W. Zhu, J. Lu, and Y.-Q. Zhang, "Video broadcasting over MIMO-OFDM systems", ISCAS, v.2, May 2003.

[11] J. Gross, J. Klaue, H. Karl, and A. Wolisz, "Subcarrier allocation for variable bit rate video streams in wireless OFDM systems," IEEE Vehicular Technology Conference, Fall. 2003.

[12] M.H. Ahmed, H. Yanikomeroglu, and S. Mahmoud, "Fairness enhancement of link adaptation techniques in wireless networks", IEEE Vehicular Technology Conference, Fall 2003.

[13] T. S. Rappaport, Wireless Communications: principles and practice: 2nd edtion, Prentice Hall, 2002. 